\title{
BMJ Open Comparison of the force applied on oral structures during intubation attempts by novice physicians between the Macintosh direct laryngoscope, Airway Scope and C-MAC PM: a high-fidelity simulator-based study
}

To cite: Nakanishi T, Shiga T, Homma $Y$, et al. Comparison of the force applied on oral structures during intubation attempts by novice physicians between the Macintosh direct laryngoscope, Airway Scope and C-MAC PM: a highfidelity simulator-based study. BMJ Open 2016;6: e011039. doi:10.1136/ bmjopen-2016-011039

- Prepublication history and additional material is available. To view please visit the journal (http://dx.doi.org/ 10.1136/bmjopen-2016011039).

Received 4 January 2016 Revised 3 May 2016 Accepted 5 May 2016

CrossMark

For numbered affiliations see end of article.

Correspondence to Dr Taizo Nakanishi; taizo81@gmail.com

\section{ABSTRACT}

Objective: We examined whether the use of Airway Scope (AWS) and C-MAC PM (C-MAC) decreased the force applied on oral structures during intubation attempts as compared with the force applied with the use of Macintosh direct laryngoscope (DL).

Design: Prospective cross-over study.

Participants: A total of 35 novice physicians participated.

Interventions: We used 6 simulation scenarios based on the difficulty of intubation and intubation devices.

Outcome measures: Our primary outcome measures were the maximum force applied on the maxillary incisors and tongue during intubation attempts, measured by a high-fidelity simulator.

Results: The maximum force applied on maxillary incisors was higher with the use of the C-MAC than with the DL and AWS in the normal airway scenario (DL, 26 Newton (N); AWS, $18 \mathrm{~N}$; C-MAC, $52 \mathrm{~N}$; $p<0.01$ ) and the difficult airway scenario (DL, $42 \mathrm{~N}$; AWS, $24 \mathrm{~N}$; C-MAC, $68 \mathrm{~N} ; \mathrm{p}<0.01)$. In contrast, the maximum force applied on the tongue was higher with the use of the DL than with the AWS and C-MAC in both airway scenarios (DL, $16 \mathrm{~N}$; AWS, $1 \mathrm{~N}$; C-MAC, $7 \mathrm{~N}$; $p<0.01$ in the normal airway scenario; DL, $12 \mathrm{~N}$; AWS, $4 \mathrm{~N}$; C$\mathrm{MAC}, 7 \mathrm{~N} ; \mathrm{p}<0.01$ in the difficult airway scenario). Conclusions: The use of C-MAC, compared with the DL and AWS, was associated with the higher maximum force applied on maxillary incisors during intubation attempts. In contrast, the use of video laryngoscopes was associated with the lower force applied on the tongue in both airway scenarios, compared with the DL. Our study was a simulationbased study, and further research on living patients would be warranted.

\section{INTRODUCTION}

Endotracheal intubation is a life-saving technique used in diverse clinical situations and
Strengths and limitations of this study

- Our study is the first to examine whether the use of video laryngoscopes decrease the force applied on oral structures during intubation attempts compared with the force applied with the use of Macintosh direct laryngoscope by novice physicians.

- As this is a simulation-based study, it might not be possible to fully extrapolate our observations to a real-world setting.

- As the participants were novice physicians, our findings may not extrapolate to intubation attempts by experienced intubators.

is one of the most frequently performed procedures in emergency departments. Physicians, including novices, need to acquire the skills to intubate confidently and safely without complications. However, the incidence of complications arising during intubation attempts remains high, despite various efforts to reduce the complications. ${ }^{1}$

While the Macintosh direct laryngoscope (DL) has been widely used for tracheal intubation, substantial evidence indicates that video laryngoscopes (VLs) play a significant role in tracheal intubation. ${ }^{2-4}$ Moreover, the use of VLs could reduce the complications associated with intubation; ${ }^{3}{ }^{5}$ previous research has demonstrated that the use of VLs leads to less force applied on oral structures during intubation attempts. ${ }^{6-8}$ In agreement with these reports, a potential mechanism by which VLs prevent complications is through the reduction of the force applied on oral structures. 
Previous studies evaluating intubation skills with VLs generally favour the use of VLs with regard to the success rate and time to intubation. ${ }^{3-11}$ For example, one study suggests even novice users achieve a higher success rate of tracheal intubation on manikins with the use of VLs compared with the DL. ${ }^{11}$ However, most of them did not quantitatively evaluate the force applied on oral structures and there is no evidence to show that novice physicians using VLs can intubate with less force applied on oral structures which, in turn, could reduce the complications associated with intubation, compared with using the DL.

To address this critical gap in knowledge, we examined whether the use of VLs (Airway Scope AWS-S100L (AWS; Pentax Corporation, Tokyo, Japan) and C-MAC PM (C-MAC; Karl Storz, Tuttlingen, Germany)) decreased the force applied on the maxillary incisors and the tongue during an intubation attempt compared with the force applied with the use of DL (figures 1 and 2).

\section{METHODS}

\section{Study design and settings}

We retrospectively analysed data from the Japanese Airway Management Quantification (JAMQ) study. The

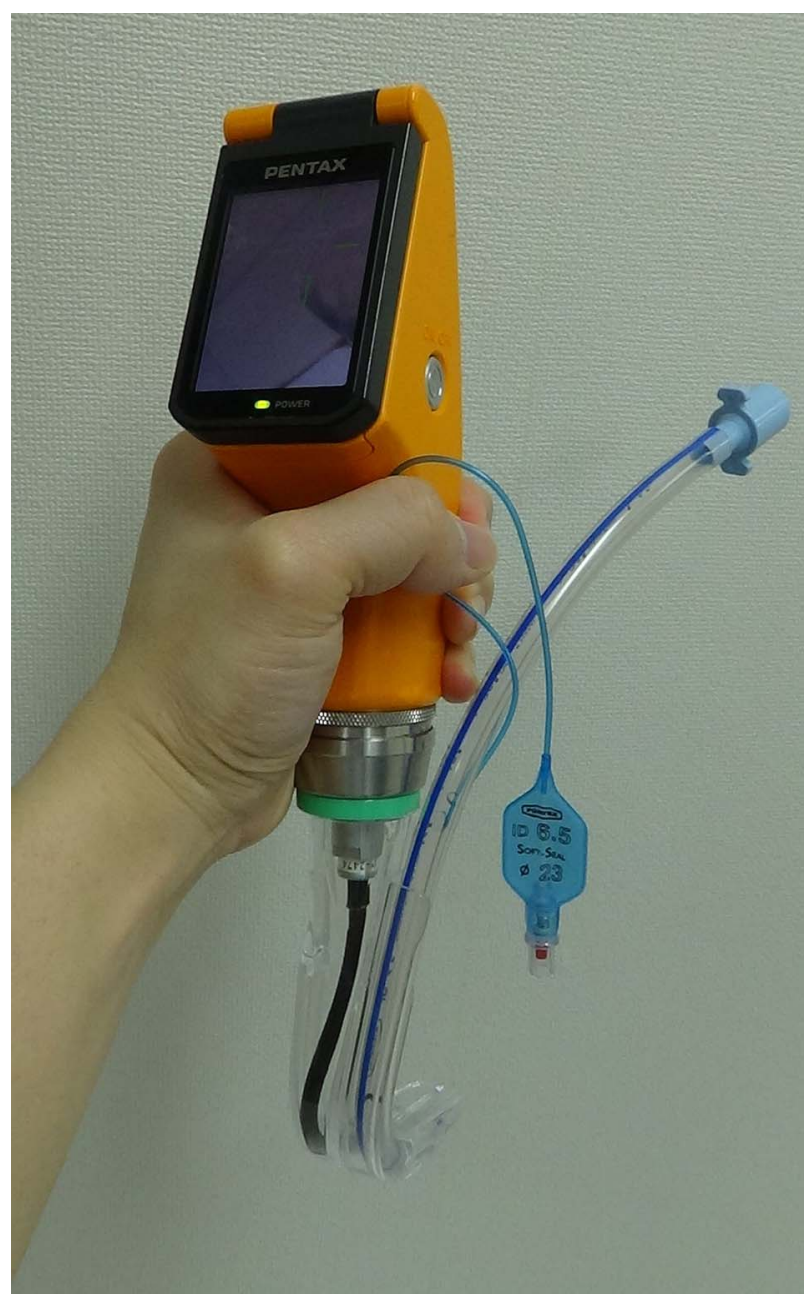

Figure 1 Photograph of Airway Scope AWS-S100L (Pentax Corporation, Tokyo, Japan).

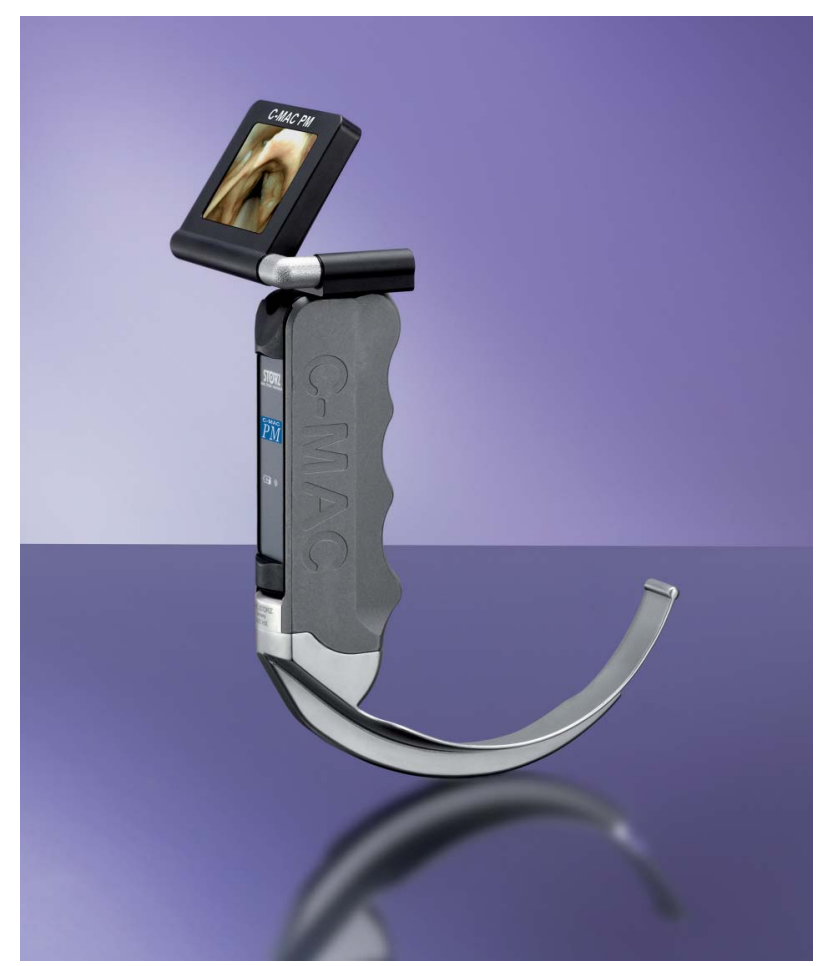

Figure 2 Photograph of C-MAC PM (Karl Storz, Tuttlingen, Germany). With permission from KARL STORZ. Endoscopy Japan K. K.

JAMQ study is a prospective multicentre data registry to investigate the factors associated with the improvement of intubation skills among novice physicians (postgraduate year (PGY) 1) using a high-fidelity simulator, with all data collection planned a priori. ${ }^{6}{ }^{12}$ The JAMQ study was initiated in April 2015 as a consortium of two academic hospitals (University of Fukui Hospital and University of Tsukuba Hospital) and two community medical centres (Fukui Prefectural Hospital and Tokyo Bay Urayasu/Ichikawa Medical Center) from different geographic regions across Japan. In the JAMQ study, we examined the intubation skills every year by using both different simulation scenarios and intubation devices. The present study utilises the data collected in April 2015 (the beginning of a new term).

\section{Data collection}

\section{Simulation scenarios}

We used data from six simulation scenarios that were based on the difficulty of intubation and specific devices (Macintosh DL, AWS and C-MAC). The simulation scenarios were as follows: (1) intubation of a normal airway using DL, (2) intubation of a difficult airway using DL, (3) intubation of a normal airway using AWS, (4) intubation of a difficult airway using AWS, (5) intubation of a normal airway using C-MAC and (6) intubation of a difficult airway using C-MAC. Size 3 and 4 blades were used appropriately for the DL and C-MAC. We defined a 'difficult airway scenario' as a scenario with a limited mouth opening to $3 \mathrm{~cm}$ (the intubation difficulty can be 
changed by limiting the mouth opening; mouth opening is $4.5 \mathrm{~cm}$ in the normal airway scenario). ${ }^{12}$ However, because the C-MAC was not available at the University of Tsukuba Hospital, four simulation courses (using the DL and AWS) were performed at the University of Tsukuba Hospital.

Prior to the simulations, all participants received a $15 \mathrm{~min}$ lecture and $15 \mathrm{~min}$ practice session to ensure that they were familiar with the proper techniques for the DL, AWS and C-MAC. Following this training, the participants of University of Fukui Hospital and Fukui Prefectural Hospital were randomly assigned to one of the three normal airway scenarios. After completion of all the normal scenarios, they sequentially participated in the other three difficult airway scenarios. Conversely, the participants of Tokyo Bay Urayasu/Ichikawa Medical Center were randomly assigned to one of the three difficult airway scenarios first and after completion of the difficult scenario they proceeded to the other three normal airway scenarios. All participants were unaware of the difficulty of intubation in all the scenarios.

\section{Study population}

Since the C-MAC was not available at the University of Tsukuba Hospital, we excluded the participants belonging to that institution. As a result, we identified 35 novice physicians from the University of Fukui Hospital, Fukui Prefectural Hospital and Tokyo Bay Urayasu/ Ichikawa Medical Center. All participants agreed to participate in this study, and written informed consent was obtained from all of them.

\section{Baseline characteristics}

Data were collected on the participants' demographics, including sex, the number of attended intubation training courses (self-reported), the number of intubations using DL (including intubation with a simulator) and the number of intubations using VLs. As the PGY 1 junior residents were novice physicians, we counted the number of intubations, including the simulation training. We used a high-fidelity airway management simulator (Waseda Kyoto Airway No.5, Kyoto-Kagaku, Kyoto,

\begin{tabular}{ll} 
Table 1 Characteristics of participants \\
\hline & $\begin{array}{l}\text { All } \\
\text { participants } \\
\mathrm{n}=35\end{array}$ \\
Characteristics & $26(74 \%)$ \\
\hline Male sex, $\mathrm{n}(\%)$ & $20(57 \%)$ \\
Number of attended intubation training course, $\mathrm{n}(\%)$ & $11(31 \%)$ \\
0 & $4(11 \%)$ \\
1 & $1(0-3)$ \\
2 & $0(0-1)$ \\
Total number of intubations, median (IQR) & \\
Total number of intubations with video & \\
laryngoscopes, median (IQR) &
\end{tabular}

Japan) to quantify the participant's intubation skills (see online supplementary figure S1). ${ }^{6}{ }^{12}$ The implanted sensors in the simulator automatically quantified the force applied on the maxillary incisors and tongue during intubation attempts.

\section{Measurement of outcomes}

Our primary outcome measures were the maximum force applied on the maxillary incisors and on the tongue during intubation attempts, measured in Newton $(\mathrm{N})$. The secondary outcome measures were time to intubation and glottic view during intubation attempts. We defined the time to intubation as the time from when the participants were ready to intubate to the successful placement of an endotracheal tube into the trachea and confirmation of ventilation. Intubation failure was defined as follows: (1) the intubator could not intubate within three intubation attempts, or (2) the intubator could not intubate within $180 \mathrm{~s}$. An intubation attempt was defined as the single insertion of a laryngoscope (DL and VLs) past the teeth. The glottic view was scored by participants using Cormack-Lehane grades. ${ }^{13}$

\section{Statistical analysis}

Since multiple scenarios were investigated and a nonnormal distribution of the outcome variables was expected, we performed Friedman's test to look for a correlation between the outcomes and devices. When a significant difference was detected in Friedman's test, we applied Wilcoxon signed-rank test with the Bonferroni correction conservatively for the post hoc analysis. When we applied the Wilcoxon signed-rank test, the $p$ value was multiplied by 3 .

All $\mathrm{p}$ values were two-sided and $\mathrm{p}$ values $<0.05$ were considered statistically significant. All data analyses were performed with EZR software V.1.28 (Saitama Medical Center, Jichi Medical University, Saitama, Japan), which is a graphical user interface for $\mathrm{R}$ (The $\mathrm{R}$ Foundation for Statistical Computing, Vienna, Austria). More precisely, it is a modified version of $\mathrm{R}$ commander designed to add statistical functions frequently used in biostatistics. $^{14}$

\section{RESULTS}

The characteristics of the participants are shown in table 1 . Of the 35 participants, 15 (42\%) had experience with intubation training courses. The overall median number of intubations was 1 (IQR 0-3), with a median of 0 (IQR $0-1$ ) among those using VLs.

All intubations were successfully performed. The maximum force (median) applied on maxillary incisors in the normal airway scenario was higher with the use of the C-MAC than with the DL and AWS (DL, $26 \mathrm{~N}$ (17$41 \mathrm{~N})$; AWS, $18 \mathrm{~N}(0-24 \mathrm{~N})$; C-MAC, $52 \mathrm{~N}(30-84 \mathrm{~N})$; $\mathrm{p}<0.01$; table 2 , figure 3 ). Similarly, the median force applied on the maxillary incisors in the difficult airway scenario was higher with the use of the C-MAC than 
with the DL and AWS (DL, $42 \mathrm{~N}(20-85 \mathrm{~N})$; AWS, $24 \mathrm{~N}$ $(17-32 \mathrm{~N})$; C-MAC, $68 \mathrm{~N}(40142 \mathrm{~N}) ; \mathrm{p}<0.01)$. In contrast, the maximum force (median) applied on the tongue in the normal airway scenario was higher with the use of the DL than with the AWS and C-MAC (DL, $16 \mathrm{~N}(8-29 \mathrm{~N})$; AWS, $1 \mathrm{~N}(0-2 \mathrm{~N})$; C-MAC, $7 \mathrm{~N}(2-18 \mathrm{~N})$; $\mathrm{p}<0.01$; figure 4$)$. Similarly, the median force applied on the tongue in the difficult airway scenario was higher with the use of the DL than with the AWS and C-MAC (DL, $12 \mathrm{~N}(7-17 \mathrm{~N})$; AWS, $4 \mathrm{~N}(2-7 \mathrm{~N})$; C-MAC, $7 \mathrm{~N}$ (4$10 \mathrm{~N}) ; \mathrm{p}<0.01)$. Median time to intubation with the DL was shorter in the normal airway scenario compared with the C-MAC (38 s (30-46s) vs $59 \mathrm{~s}$ (44-80 s); $\mathrm{p}<0.01)$. In the difficult scenario, time to intubation was somewhat shorter in the DL group than the other groups, but was not statistically significant. In the Cormack-Lehane grade score, no significant differences were found among any of the devices in either the normal or difficult airway scenarios.

For the DL, the force applied on maxillary incisors was significantly higher in the difficult airway scenario compared with that in the normal airway scenario $(26 \mathrm{~N}$ $(17-41 \mathrm{~N})$ vs $42 \mathrm{~N}(20-85 \mathrm{~N}) ; \mathrm{p}<0.01$; see online supplementary table S1). Conversely, the force applied on the tongue was significantly higher in the normal airway scenario compared with that in the difficult airway scenario $(16 \mathrm{~N}(8-29 \mathrm{~N})$ vs $12 \mathrm{~N}(7-17 \mathrm{~N}) ; \mathrm{p}<0.01)$. Time to intubation for the DL was significantly shorter in the normal airway scenario than in the difficult airway scenario (38 s (30-46 s) vs $49 \mathrm{~s}(36-70 \mathrm{~s})$; $\mathrm{p}<0.01)$.

For the AWS, the only significant difference was found in the force applied on the tongue; the force applied on the tongue was higher in the difficult airway scenario than in the normal airway scenario $(1 \mathrm{~N}(0-2 \mathrm{~N})$ vs $4 \mathrm{~N}$
$(2-7 \mathrm{~N}) ; \mathrm{p}<0.01)$. Similarly, for the C-MAC, the only significant difference was found in the force applied on the maxillary incisors; the force applied on the maxillary incisors was higher in the difficult airway scenario than in the normal airway scenario $(52 \mathrm{~N}(30-84 \mathrm{~N})$ vs $68 \mathrm{~N}$ $(40-142 \mathrm{~N}) ; \mathrm{p}<0.01)$

\section{DISCUSSION}

In this multicentre cross-over study, we found that the use of the C-MAC, in comparison to the DL and AWS, by novice physicians was associated with higher maximum force applied on the maxillary incisors during intubation attempts in the normal and difficult airway scenarios. Additionally, we found that the use of the DL was associated with higher maximum force applied on the tongue in both airway scenarios, compared with the VLs. To the best of our knowledge, our study is the first to report that intubation with the AWS and C-MAC by novice physicians can reduce the force applied on the tongue. However, our study was simulation based, so it requires cautious interpretation to extrapolate these results to living patients.

Dental injury is one of the frequent adverse events of intubation and the most common claim against intubators. ${ }^{15}$ Previous research has shown that the complete arch of the incisors has maximum bite force ranging from 150 to $200 \mathrm{~N} .{ }^{16}$ Based on this information, the peak force on the maxillary incisors of more than 150 $200 \mathrm{~N}$ might be a risk factor for dental injury during intubation attempts. Although some studies have reported that the use of VLs (ie, GlideScope, McGrath and V-MAC) were associated with less force applied on maxillary incisors, ${ }^{8} 17$ our results showed the applied

Table 2 Comparisons of Macintosh direct laryngoscope, AWS and C-MAC PM for intubation

\begin{tabular}{|c|c|c|c|c|c|c|}
\hline \multirow[b]{2}{*}{ Outcomes } & \multirow[b]{2}{*}{$\begin{array}{l}D L \\
n=35\end{array}$} & \multicolumn{2}{|l|}{$\begin{array}{l}\text { AWS } \\
n=35\end{array}$} & \multicolumn{2}{|l|}{$\begin{array}{l}\text { C-MAC } \\
n=35\end{array}$} & \multirow{2}{*}{$\begin{array}{l}\text { p Value } \\
\text { (comparison } \\
\text { among } 3 \\
\text { groups) }\end{array}$} \\
\hline & & & $\begin{array}{l}\text { p Value } \\
\text { (comparison } \\
\text { for } D L \text { ) }\end{array}$ & & $\begin{array}{l}\text { p Value } \\
\text { (comparison } \\
\text { for } D L \text { ) }\end{array}$ & \\
\hline \multicolumn{7}{|c|}{ Force on maxillary incisors $(\mathrm{N})$} \\
\hline Normal airway & $26(17-41)$ & $18(0-24)^{*}$ & 0.06 & $52(30-84)$ & $<0.01$ & $<0.01$ \\
\hline Difficult airway & $42(20-85)$ & $24(17-32)^{*}$ & 0.01 & $68(40-142)$ & $<0.01$ & $<0.01$ \\
\hline \multicolumn{7}{|c|}{ Applied force on the tongue $(\mathrm{N})$} \\
\hline Normal airway & $16(8-29)$ & $1(0-2)^{*}$ & $<0.01$ & $7(2-18)$ & $<0.01$ & $<0.01$ \\
\hline Difficult airway & $12(7-17)$ & $4(2-7) \dagger$ & $<0.01$ & $7(4-10)$ & 0.03 & $<0.01$ \\
\hline \multicolumn{7}{|c|}{ Time to intubation (seconds) } \\
\hline Normal airway & $38(30-46)$ & $48(38-63)$ & 0.12 & $59(44-80)$ & $<0.01$ & $<0.01$ \\
\hline Difficult airway & $49(36-70)$ & $50(40-62)$ & 0.99 & $66(43-95)$ & 0.13 & 0.04 \\
\hline \multicolumn{7}{|c|}{ Cormack-Lehane grades } \\
\hline Normal airway & $1(1-2)$ & $1(1-1)$ & $\ddagger$ & $1(1-2)$ & $\ddagger$ & 0.10 \\
\hline Difficult airway & $2(1-2)$ & $1(1-2)$ & $\ddagger$ & $1(1-2)$ & $\ddagger$ & 0.08 \\
\hline \multicolumn{7}{|c|}{$\begin{array}{l}\text { Data are expressed as median (IQR). } \\
{ }^{*} p<0.01 \text { compared with C-MAC. } \\
t p<0.05 \text { compared with C-MAC. } \\
\text { †There were no significant differences among the three groups. } \\
\text { AWS, Airway Scope; C-MAC, C-MAC PM; DL, Macintosh direct laryngoscope; N, newton. }\end{array}$} \\
\hline
\end{tabular}


Figure 3 Comparison of forces applied on maxillary incisors in the normal and difficult airway scenarios with the use of DL, AWS and C-MAC. Box and whisker graph indicating median, upper and lower quartiles, outliers and extreme values. DL, Macintosh direct laryngoscope; AWS, Airway Scope; C-MAC, C-MAC PM.

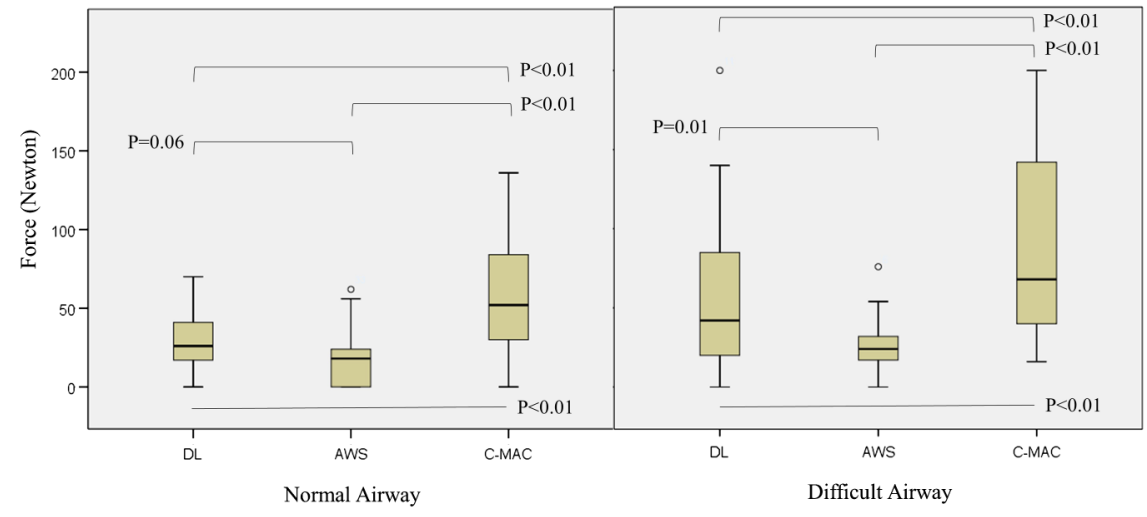

force was higher in the C-MAC group. One plausible explanation for this disparity is the lack of participants' intubation experiences. Since the participants were novice physicians, they were more likely to observe the monitor when the C-MAC passed the teeth. However, the force applied on maxillary incisors in the C-MAC group did not exceed the maximum bite force in normal and difficult airway scenarios. This might, thus, suggest even novice physicians can intubate with C-MAC safely in terms of dental injury.

In contrast, the use of AWS, also a VL, was not associated with the higher force applied on the maxillary incisors compared with the DL. One may surmise that the lower force with the use of AWS was attributable to the structural differences between the AWS and DL. The AWS is a unique device that does not require an alignment of the oral, pharyngeal and tracheal axes in one line to obtain glottic view during intubation attempts. ${ }^{18}$ Therefore, the participants could insert the AWS through the oropharyngeal space by focusing on the teeth without watching the monitor.

Our results also demonstrated that the use of VLs was associated with lower force applied on the tongue, which is consistent with previous studies. 71920 Excessive force on the oral structures can lead to pathological responses from the cardiovascular and respiratory systems, such as bradycardia and cardiopulmonary arrest. Thus, tracheal intubation should be performed with minimal force to reduce the number of adverse events. Further, it is important to note that the haemodynamic response to intubation attempts might be less with VLs because of the reduced force on the oral structures. $^{21} 22$ In the current study, the use of AWS and C-MAC reduced the force applied to the tongue. The use of VLs enabled intubations without any excessive effort exerted to achieve a direct line of sight as compared with the DL. Taken together, the use of VLs reduces the force applied to the tongue, and thus may decrease the risk of a change in haemodynamics during intubation attempts.

Although VLs are likely to be beneficial compared with the DL, novice physicians should be well acquainted with the unique characteristics of each VL. Senior physicians also need to know the characteristics and educate younger physicians on the appropriate use of VLs based on these characteristics.

\section{LIMITATIONS}

Our study has several potential limitations. First, as the participants were novice physicians, our findings may not extrapolate to intubation attempts by experienced intubators. However, our study was not subject to differences in intubation experience (experienced intubators are likely to use their preferable intubation device). Therefore, our results solely demonstrated the difference in intubation devices. Second, we quantified the force applied to the maxillary incisors and the tongue.

Figure 4 Comparison of forces applied on the tongue in the normal and difficult airway scenarios with the use of DL, AWS and C-MAC. Box and whisker graph indicating median, upper and lower quartiles, outliers and extreme values. DL, Macintosh direct laryngoscope; AWS, Airway Scope; C-MAC, C-MAC PM.

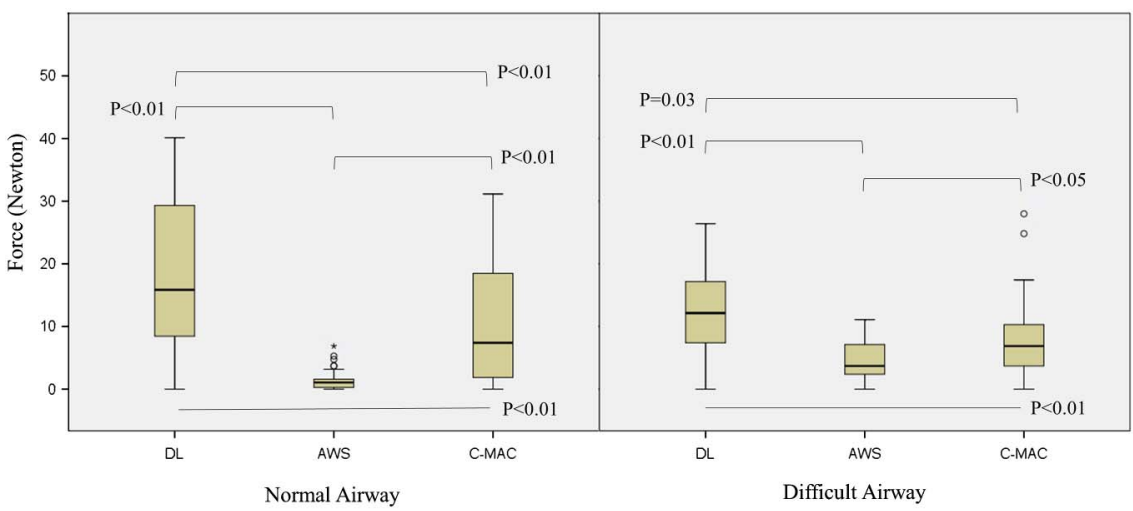


Although applied force to the oral structures may be associated with adverse events, including dental injury and haemodynamic stress, we did not directly demonstrate the association between intubation devices and adverse events. Third, we did not calculate the sample size in advance because this study was conducted retrospectively using part of the prospective multicentre data registry. However, the post hoc power calculation demonstrates the power is sufficient (power $>0.90$ ) in all primary outcomes. Finally, this was a simulation-based study; therefore, our results might not necessarily coincide with results in living patients. For example, in clinical settings, various factors, such as cardiopulmonary resuscitation, bleeding and secretions, might interrupt intubation attempts. Also evidence shows that there is a difference in anatomical structure of manikins compared with humans. Therefore, there should be a cautious interpretation of our results. ${ }^{23}$

\section{CONCLUSIONS}

In this multicentre cross-over study, we found that the use of C-MAC, compared with DL and AWS, was associated with the higher maximum force applied on maxillary incisors during intubation attempts in normal and difficult airway scenarios. In contrast, we also found that the use of VLs was associated with lower force applied on the tongue in normal and difficult airway scenarios, compared with DL. Although VLs are beneficial for novice physicians in intubation, educators should be acquainted with the characteristics of intubation devices and instruct novice physicians on the appropriate use of each intubation device. Our study was a simulation-based study, and further research on living patients would be warranted.

\section{Author affiliations}

${ }^{1}$ Department of Emergency Medicine, Fukui Prefectural Hospital, Fukui, Japan ${ }^{2}$ Department of Emergency Medicine, Tokyo Bay Urayasu/Ichikawa Medical Center, Noguchi Hideyo Memorial International Hospital, Urayasu, Japan ${ }^{3}$ Department of Emergency and Critical Care Medicine, University of Tsukuba Hospital, Tsukuba, Japan

${ }^{4}$ Department of Emergency Medicine, Massachusetts General Hospital, Boston, Massachusetts, USA

Contributors TN contributed to study concept and design, acquisition of the data, analysis and interpretation of the data, and drafting of the manuscript. TS contributed to study concept and design, critical revision of the manuscript, and study supervision. YH contributed to study concept and design, acquisition of the data, and analysis and interpretation of the data. YK contributed to study concept and design, acquisition of the data, and analysis and interpretation of the data. TG contributed to study concept and design, critical revision of the manuscript, statistical expertise, and study supervision.

Funding This research received no specific grant from any funding agency in the public, commercial or not-for-profit sectors.

Competing interests None declared.

Ethics approval Institutional Review Board of University of Fukui, University of Tsukuba Ethics Committee, Ethics Committee of Fukui Prefectural Hospital and Ethics Committee of Tokyo Bay Urayasu/lchikawa Medical Center.

Provenance and peer review Not commissioned; externally peer reviewed.
Data sharing statement No additional data are available.

Open Access This is an Open Access article distributed in accordance with the Creative Commons Attribution Non Commercial (CC BY-NC 4.0) license, which permits others to distribute, remix, adapt, build upon this work noncommercially, and license their derivative works on different terms, provided the original work is properly cited and the use is non-commercial. See: http:// creativecommons.org/licenses/by-nc/4.0/

\section{REFERENCES}

1. Jaber $\mathrm{S}$, Jung $\mathrm{B}$, Corne $\mathrm{P}$, et al. An intervention to decrease complications related to endotracheal intubation in the intensive care unit: a prospective, multiple-center study. Intensive Care Med 2010;36:248-55.

2. Sakles JC, Mosier J, Chiu S, et al. A comparison of the C-MAC video laryngoscope to the Macintosh direct laryngoscope for intubation in the emergency department. Ann Emerg Med 2012;60:739-48.

3. Malik MA, Hassett P, Carney J, et al. A comparison of the Glidescope, Pentax AWS, and Macintosh laryngoscopes when used by novice personnel: a manikin study. Can $J$ Anesth 2009;56:802-11.

4. Paolini JB, Donati F, Drolet P. Review article: video-laryngoscopy: another tool for difficult intubation or a new paradigm in airway management? Can J Anaesth 2013;60:184-91.

5. Mosier J, Whitmore S, Bloom J, et al. Video laryngoscopy improves intubation success and reduces esophageal intubations compared to direct laryngoscopy in the medical intensive care unit. Crit Care 2013;17:R237.

6. Goto T, Koyama Y, Kondo T, et al. A comparison of the force applied on oral structures during intubation attempts between the Pentax-AWS airwayscope and the Macintosh laryngoscope: a high-fidelity simulator-based study. BMJ Open 2014;4: e006416.

7. Carassiti M, Zanzonico R, Cecchini S, et al. Force and pressure distribution using Macintosh and GlideScope laryngoscopes in normal and difficult airways: a manikin study. $\mathrm{Br} J$ Anaesth 2012;108:146-51

8. Lee RA, van Zundert AA, Maassen RL, et al. Forces applied to the maxillary incisors during video-assisted intubation. Anesth Analg 2009;108:187-91

9. Fukuda T, Sugimoto $\mathrm{Y}$, Yamashita S, et al. Forces applied to the maxillary incisors during tracheal intubation and dental injury risks of intubation by beginners: a manikin study. Acta Anaesthesiol Taiwan 2011;49:12-15.

10. Aziz M, Dillman D, Kirsch JR, et al. Video laryngoscopy with the Macintosh video laryngoscope in simulated prehospital scenarios by paramedic students. Prehosp Emerg Care 2009;13:251-5.

11. Ray DC, Billington C, Kearns PK, et al. A comparison of McGrath and Macintosh laryngoscopes in novice users: a manikin study. Anaesthesia 2009;64:1207-10.

12. Yohan $\mathrm{N}$, Wang $\mathrm{C}$, Tokumoto $\mathrm{M}$, et al. Development of airway management training system WKA-4: provide useful feedback of trainee performance to trainee during airway management. International Conference on Complex Medical Engineering; 2012.

13. Cormack RS, Lehane J. Difficult tracheal intubation in obstetrics. Anaesthesia 1984;39:1105-11.

14. Kanda $Y$. Investigation of the freely available easy-to-use software 'EZR' for medical statistics. Bone Marrow Transplant 2013;48: 452-8.

15. Gaudio RM, Feltracco P, Barbieri S, et al. Traumatic dental injuries during anaesthesia: part I: clinical evaluation. Dent Traumatol 2010;26:459-65.

16. Fontijn-Tekamp FA, Slagter AP, Van Der Bilt A, et al. Biting and chewing in overdentures, full dentures, and natural dentitions. J Dent Res 2000;79:1519-24.

17. Lee RA, van Zundert AA, Maassen RL, et al. Forces applied to the maxillary incisors by video laryngoscopes and the Macintosh laryngoscope. Acta Anaesthesiol Scand 2012; 56:224-9.

18. Koyama $\mathrm{Y}$, Nishihama M, Inagawa G, et al. Comparison of haemodynamic responses to tracheal intubation using the Airway

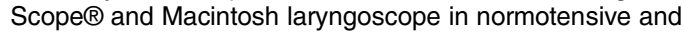
hypertensive patients. Anaesthesia 2011;66:895-900.

19. Gaszynski TM. Forces applied by the laryngoscope blade onto the tongue during intubation attempts: a comparison between Mackintosh, AirTraq and Pentax AWS in a mannequin study. Eur J Anaesthesiol 2011;28:463-4. 
20. Russell T, Khan S, Elman J, et al. Measurement of forces applied during Macintosh direct laryngoscopy compared with

GlideScope $®$ videolaryngoscopy. Anaesthesia 2012;67:626-31.

21. Dashti M, Amini S, Azarfarin R, et al. Hemodynamic changes following endotracheal intubation with glidescope ${ }^{\circledR}$ video-laryngoscope in patients with untreated hypertension. Res Cardiovasc Med 2014;3 e17598.
22. Lee $\mathrm{H}$. The Pentax airway scope versus the Macintosh laryngoscope: comparison of hemodynamic responses and concentrations of plasma norepinephrine to tracheal intubation. Korean J Anesthesiol 2013;64:315-20.

23. Schebesta K, Hüpfl M, Rössler B, et al. Degrees of reality: airway anatomy of high-fidelity human patient simulators and airway trainers. Anesthesiology 2012;116:1204-9. 